\title{
Endocan: A Biomarker for Hepatosteatosis in Patients with Metabolic Syndrome
}

\author{
Hande Erman $\mathbb{D}^{1},{ }^{1}$ Engin Beydogan, ${ }^{2}$ Seher Irem Cetin, ${ }^{3}$ and Banu Boyuk $\mathbb{D}^{3}$ \\ ${ }^{1}$ Department of Internal Medicine, SBU Fatih Sultan Mehmet Education and Research Hospital, Turkey \\ ${ }^{2}$ Department of Radiology, SBU Taksim Education and Research Hospital, Turkey \\ ${ }^{3}$ Department of Internal Medicine, SBU Taksim Education and Research Hospital, Turkey \\ Correspondence should be addressed to Hande Erman; handeerman@yahoo.com
}

Received 24 December 2019; Revised 19 February 2020; Accepted 9 March 2020; Published 1 April 2020

Academic Editor: Agnieszka Dobrzyn

Copyright () 2020 Hande Erman et al. This is an open access article distributed under the Creative Commons Attribution License, which permits unrestricted use, distribution, and reproduction in any medium, provided the original work is properly cited.

Background. Nonalcoholic fatty liver disease (NAFLD) is one of the most common chronic liver diseases, which has recently been mentioned as an independent cardiovascular risk factor. Objectives. Endocan is a novel molecule of endothelial dysfunction. We aimed to evaluate the associations of serum endocan levels with the hepatic steatosis index (HSI), fatty liver index (FLI), and degrees of hepatosteatosis in patients with metabolic syndrome with NAFLD. Design and Setting. This cross-sectional prospective study was performed in the outpatient clinic of an internal medicine department. Methods. The study included 40 patients with metabolic syndrome with NAFLD as noted using hepatic ultrasound and 20 healthy controls. Secondary causes of fatty liver were excluded. FLI and HSI calculations were recorded. Serum endocan level values were obtained after overnight fasting. Results. Higher values of HSI and FLI were found in the NAFLD groups than in the control groups $(p<0.001)$. Five $(12.5 \%)$ of 20 patients with liver steatosis had grade 1 liver steatosis, 15 (37.5\%) patients had grade 2 liver steatosis, and 20 (50\%) patients had grade 3 liver steatosis. Serum endocan levels were lower in patients with NAFLD compared with the healthy controls $(146.56 \pm 133.29 \mathrm{pg} / \mathrm{mL}$ vs. $433.71 \pm 298.01 \mathrm{pg} / \mathrm{mL}, p<0.001)$. ROC curve analysis suggested that the optimum endocan value cutoff point for NAFLD was $122.583 \mathrm{pg} / \mathrm{mL}$ (sensitivity: 71.79\%, specificity: 90\%, PPV: 93.3\%, and NPV: 62.1\%). Conclusion. Serum endocan concentrations are low in patients with NAFLD, and the optimum cutoff point is $122.583 \mathrm{pg} / \mathrm{mL}$. HSI and FLI were higher in patients with NAFLD; however, there was no correlation with serum endocan.

\section{Introduction}

Metabolic syndrome is an increasing public health problem for developed countries. The shared pathogenic mechanisms between metabolic syndrome and obesity-related disorders force us to be more sensitive in analyzing this population due to potential cardio metabolic consequences [1].

Nonalcoholic fatty liver disease (NAFLD) has a prevalence of $25 \%$ worldwide and is described as the leading cause of chronic liver disease [2]. In a study based on the data of NHANES III, liver disease was found to be the third leading cause of death among persons with NAFLD [3]. Its prevalence is increasing in conjunction with metabolic syndrome due to engaged risk factors, which include high body mass index and abdominal obesity, type 2 diabetes, dyslipidemia (high triglycerides and/or low high-density-lipoproteins), age, male sex, and alcohol consumption [4-6].

NAFLD is a silent condition that encompasses nonalcoholic fatty liver (NAFL) and nonalcoholic steatohepatitis (NASH) [7]. Although ultrasound and magnetic resonance are helpful for the diagnosis, liver biopsy is still the gold standard [8]. New insights and diagnostic improvements in NAFLD such as transient elastography and FibroScan are exciting alternatives [9]. However, we targeted focusing on noninvasive, cheap, and useful biomarkers in clinical practice. In this way, the role of circulating biomarkers related to endothelial dysfunction and the severity of underlying liver disease need to be investigated. Thus, we tested circulating levels of serum endocan to determine whether they may improve the timely prediction of fat content of liver and NAFLD. 
Endocan, previously known as endothelial cell specific molecule-1, is a soluble form of dermatan sulfate, which is expressed by vascular endothelial cells of the lung, liver, and kidney. Tumor necrosis factor, interleukin-1, and lipopolysaccharides are suggested regulators of endocan expression $[10,11]$. Systemic inflammation and subclinical atherosclerosis have a trigger role in atherosclerotic cardiovascular disease and are associated with NAFLD [12].

In the light of data in the literature, only a minority of patients with NAFLD proceed to the fibrotic and cirrhotic stages and develop end-stage liver disease. However, it is already known that nearly $40 \%$ of patients with NAFLD die of cardiovascular complications. For this reason, our study targeted predicting the stage and presence of NAFLD using serum endocan levels in order to prevent associated risks as soon as possible.

\section{Methods}

2.1. Study Population. In this cross-sectional study, 40 patients with metabolic syndrome with NAFLD were assessed. The criteria used for the diagnosis of metabolic syndrome were those recommended by the National Cholesterol Education Program. Expert Panel on Detection, Evaluation, and Treatment of High Blood Cholesterol in Adults-Adult Treatment Panel III (NCEP-ATP III), which is defined as the presence of at least three of these components: (1) increased waist circumference $(>102 \mathrm{~cm}$ for men and $>88 \mathrm{~cm}$ for women), (2) elevated triglycerides ( $\geq 150 \mathrm{mg} / \mathrm{dL}$ ) or use of triglyceride-lowering drugs, (3) low levels of high-density lipoprotein (HDL) cholesterol ( $<40 \mathrm{mg} / \mathrm{dL}$ in men and $<50 \mathrm{mg} / \mathrm{dL}$ in women), (4) hypertension $(\geq 130 / \geq 85 \mathrm{mmHg})$ or use of antihypertensive drugs, and (5) fasting glucose $(\geq 110 \mathrm{mg} / \mathrm{dL})$ or use of antidiabetic drugs [13].

Patients were included if they had a diagnosis of NAFLD as noted using hepatic ultrasound. Participants with any of the following possible secondary causes of fatty liver were excluded from the current analyses: (1) excessive alcohol intake (alcohol consumption was $\geq 20 \mathrm{~g} /$ day for men and $\geq 10 \mathrm{~g}$ /day for women over the past 12 months), (2) positive antihepatitis $\mathrm{C}$ virus (HCV) or hepatitis B surface antigen (HBsAg), (3) history drug use for treating fatty liver (i.e., amiodarone, corticosteroids, methotrexate, or tamoxifen), or (4) the absence of other causes of liver dysfunction, such as autoimmune liver disease, primary sclerosing cholangitis, Wilson's disease, and hereditary hemochromatosis. Other exclusion criteria were the presence of ischemic heart disease, congenital heart disease, valvular heart disease, and neoplastic, inflammatory, and infectious diseases.

This study was performed according to the guidelines of the Declaration of Helsinki, and it was approved by the ethics review committee of our hospital.

2.2. Physical and Laboratory Measurements. Anthropometric measurements, including weight, height, and systolic/diastolic blood pressure (BP), were measured following standardized protocols. Participants' seated BP was measured twice every $5 \mathrm{~min}$ on the right arm after $5 \mathrm{~min}$ of rest with a sphyg- momanometer. The mean of the two readings was used in data analysis. The body mass index (BMI) was calculated according to the weight $(\mathrm{kg})$ divided by the square of height (meters). Waist circumflex (WC) was measured midway between the uppermost border of the iliac crest and the lower border of the costal margin.

Overnight fasting (at least $8 \mathrm{~h}$ ) blood samples were collected from the antecubital vein of each individual. Biochemical measurements, including assessment of fasting plasma glucose, total cholesterol (TCH), triglycerides (TG), lowdensity lipoprotein cholesterol (LDL-C), HDL-C, alanine aminotransferase (ALT), aspartate aminotransferase (AST), gamma-glutamyltransferase (GGT), and glycated hemoglobin (HbA1C), were measured enzymatically on an auto analyzer (COBAS 311, Roche Diagnostics GmbH, Mannheim, Germany). Particle-enhanced immunoturbidimetry was performed using a Behring Nephelometer BN-100 (Behring Diagnostic, Frankfurt, Germany) which was used to measure C-reactive protein (CRP). The sensitivity of the test was $0.1 \mathrm{mg} / \mathrm{L}$. Serum endocan levels were measured using a Sunred enzyme-linked immunosorbent assay (ELISA) kit (Sunred Biological Technologies Human ECSM1/ENDOCAN ELISA Kit, catalog No.: 201-12-1978, China). The intra- and interassay variabilities of the ELISA kit were $5.1 \%$ and $6.1 \%$, respectively. The minimum detectable level of endocan was $31.2 \mathrm{pg} / \mathrm{mL}$.

\subsection{FLI and HSI Were Calculated on the Basis Sample Analyses.} Hepatic steatosis index $(\mathrm{HSI})=8 *$ ALT/AST + BMI $(+2$, if $\mathrm{DM} ;+2$, if female). At a value of $<30.0$, HSI could rule out steatosis. At a value of $\geq 36$, HSI could rule in steatosis [14].

Fatty liver index $(\mathrm{FLI})=\operatorname{logistic}(0.953 * \ln (\mathrm{TG})+0.139 *$ $\mathrm{BMI}+0.718+\ln (\mathrm{GGT})+0.053 *$ waist 15.745$) * 100$, where logistic $(\mathrm{x})=1 /(1+\mathrm{ex})$ denotes the logistic function and $\ln$ the natural logarithm. Values $<30$ rule out steatosis, and values $\geq 60$ rule in steatosis [15].

2.4. Liver Ultrasound Measurements. Ultrasound evaluations were performed by a single radiologist using a 1-6 MHz PVT375 BT convex transducer (Toshiba A500 Platinum). Ultrasound examination of the liver was performed after 12 hours fasting. Each subject was examined in the supine and left lateral positions during quiet inspiration and asked to stop breathing during inspiration. The presence or absence and grading of fatty infiltration of the liver were recorded. Hepatic steatosis was defined as the presence of an ultrasonography pattern of parenchymal brightness (from normal to severe increased), liver-kidney contrast (absent $=0 /$ present $=1$ ), deep beam attenuation (diaphragm bright and clear $=0$ /diaphragm blurred $=1$ ), and bright vessel walls in the parenchyma (present $=0 /$ absent $=1$ ) [16].

2.5. Statistical Analysis. Frequency, ratio, mean, minimum, maximum, and standard deviation values were used in the descriptive statistics to determine continuous variables. Student's $t$-test was used for comparisons of two independent and normally distributed variables. The Mann-Whitney $U$ test was performed for the comparison of independent and nonnormally distributed variables. The chi-squared test and 
TABLE 1: Clinical and laboratory characteristics of controls and patients of NAFLD.

\begin{tabular}{|c|c|c|c|}
\hline & $\begin{array}{c}\text { Patients of NAFLD }(n=40) \\
\text { Mean } \pm \text { s.d. } / n, \%\end{array}$ & $\begin{array}{l}\text { Control group }(n=20) \\
\text { Mean } \pm \text { s.d. } / n, \%\end{array}$ & \\
\hline Age (years) & $54.05 \pm 9.09$ & $52.5 \pm 7.98$ & \\
\hline \multicolumn{4}{|l|}{ Gender } \\
\hline Male & $16(40 \%)$ & $10(50 \%)$ & \\
\hline Female & $24(60 \%)$ & $10(50 \%)$ & \\
\hline BMI $\left(\mathrm{kg} / \mathrm{m}^{2}\right)$ & $31.62 \pm 4.65$ & $25.58 \pm 3.67$ & $* *$ \\
\hline Waist circumference $(\mathrm{cm})$ & $109.72 \pm 11.73$ & $85.05 \pm 11.79$ & $* *$ \\
\hline Systolic pressure (mmHg) & $132.05 \pm 21.08$ & $117.5 \pm 9.25$ & $* *$ \\
\hline Diastolic pressure (mmHg) & $73.97 \pm 11.99$ & $71.25 \pm 8.72$ & \\
\hline Fasting blood glucose (mg/dL) & $171.59 \pm 96.09$ & $91.2 \pm 12.48$ & ** \\
\hline HbAlc (\%) & $8.42 \pm 2.76$ & $5.55 \pm 0.46$ & $* *$ \\
\hline Triglyceride (mg/dL) & $250.05 \pm 198.03$ & $115.1 \pm 57.96$ & ** \\
\hline Total cholesterol (mg/dL) & $211.1 \pm 49.91$ & $207.1 \pm 35.89$ & \\
\hline $\mathrm{LDL}(\mathrm{mg} / \mathrm{dL})$ & $119.56 \pm 36.27$ & $129.1 \pm 30.31$ & \\
\hline HDL (mg/dL) & $46.1 \pm 10.81$ & $54.95 \pm 14.92$ & $*$ \\
\hline CRP (mg/L) & $6.1 \pm 3.87$ & $2.3 \pm 2.02$ & ** \\
\hline AST (U/L) & $27.38 \pm 17.34$ & $19.75 \pm 3.67$ & \\
\hline $\operatorname{ALT}(\mathrm{U} / \mathrm{L})$ & $32.49 \pm 25.53$ & $19.05 \pm 5.92$ & $*$ \\
\hline GGT (U/L) & $40.33 \pm 31.72$ & $20.6 \pm 8.59$ & $* *$ \\
\hline Endocan (pg/mL) & $146.56 \pm 133.29$ & $433.71 \pm 298.01$ & ** \\
\hline FLI & $51.52 \pm 24.7$ & $6.57 \pm 35.23$ & $* *$ \\
\hline HSI & $33.9 \pm 29.6$ & $17.6 \pm 7.1$ & ** \\
\hline \multicolumn{4}{|l|}{ NAFLD grade } \\
\hline 0 & $0(0 \%)$ & $20(100 \%)$ & \\
\hline 1 & $5(12.5 \%)$ & $0(0 \%)$ & \\
\hline 2 & $15(37.5 \%)$ & $0(0 \%)$ & \\
\hline 3 & $20(50.0 \%)$ & $0(0 \%)$ & \\
\hline
\end{tabular}

Mann-Whitney $U$, Student's $t$, and Fisher's exact test. Statistical significance: ${ }^{*} p<0.05,{ }^{* *} p<0.01$. BMI: body mass index; HOMA-IR: homeostasis model assessment of insulin resistance; LDL: low-density lipoprotein; HDL: high-density lipoprotein; CRP: C-reactive protein; AST: aspartate transaminase; ALT: alanine transaminase; GGT: gamma-glutamyl transpeptidase; FLI: fatty liver index; HSI: hepatic steatosis index.

Fisher's exact test were performed to determine differences between categorical variables. Multiple linear logistic regression analysis was performed to determine the effect levels of the parameters. Spearman's correlation tests were used for correlation analyses. Receiving operating characteristics (ROC) curve analysis was performed to define the sensitivity and specificity of serum endocan to predict NAFLD. Statistical significance was assessed at $p<0.05$. Statistical analysis was performed using the MedCalc Statistical Software version 12.7.7 (MedCalc Software bvba, Ostend, Belgium; http://www.medcalc.org; 2013).

\section{Results}

The clinical characteristics of the study subjects are shown in Table 1. Sex and age distribution were similar between the groups. There were no significant differences in DBP, TCH, LDL-C, and AST levels between the patients with metabolic syndrome with NAFLD and control subjects.
The patients with NAFLD had a significantly higher SBP, BMI, waist circumference, fasting plasma glucose, HbA1C, TG, ALT, GGT, and CRP levels than the control subjects $(p<0.01)$. It was observed that HDL-C levels were significantly lower in patients with NAFLD than in the controls $(p<0.05)$. The serum endocan levels were significantly lower in patients with NAFLD compared with the healthy controls $(146.56 \pm 133.29 \mathrm{pg} / \mathrm{mL}$ vs. $433.71 \pm 298.01 \mathrm{pg} / \mathrm{mL}$, $p<0.001)$. The HSI and FLI values were statistically significantly higher in the NAFLD groups than in the control group $(p<0.001)$.

Five $(12.5 \%)$ of 20 patients with liver steatosis had grade 1 liver steatosis, $15(37.5 \%)$ patients had grade 2 liver steatosis, and $20(50.0 \%)$ patients had grade 3 liver steatosis.

The serum endocan levels showed a positive correlation with age $(r=0.309 ; p=0.05)$ and a negative correlation with the BMI $(r=-0.386 ; p<0.015)$ in NAFLD, as shown in Table 2. However, there was no significant correlation between the other variables and endocan levels. Regression 
TABLE 2: Correlation between endocan and characteristics in patients of NAFLD.

\begin{tabular}{lc}
\hline & Endocan \\
\hline $\begin{array}{l}\text { Age (years) } \\
r\end{array}$ & 0.309 \\
$p$ & 0.05 \\
BMI $\left(\mathrm{kg} / \mathrm{m}^{2}\right)$ & \\
$r$ & -0.386 \\
$p$ & 0.015 \\
HSI & \\
$r$ & -0.223 \\
$p$ & 0.173 \\
FLI & \\
$r$ & -0.262 \\
$p$ & 0.112 \\
\hline NAFLD: nonalcoholic fatty liver disease; BMI: body mass index; HSI: hepatic \\
steatosis index; FLI: fatty liver index.
\end{tabular}

analysis of BMI and serum endocan revealed that exponentiated coefficient was found to be -40.43 which means one unit increase of BMI results in $40.43 \%$ decrease of serum endocan (not shown in table).

ROC analysis and diagnostic screening tests were used to determine the cutoff point for endocan. Patients who had an endocan level lower than $122.583 \mathrm{pg} / \mathrm{mL}$ were indicated NAFLD with a sensitivity of $71.79 \%$ and a specificity of $90 \%$. The cutoff value of serum endocan level to predict NAFLD was $122.583 \mathrm{pg} / \mathrm{mL}$ with a positive predictive value of $93.3 \%$ and a negative predictive value of $62.1 \%$. The cutoff value of HSI was 16.76 to predict NAFLD (AUC $=0.893, p<0.001$ ). The cutoff value of FLI was 40.86 (AUC $=0.893, p<0.001$ ) (Table 3 and Figure 1 ). Furthermore, AUC values for endocan and HSI were similar $(p=0.219)$. Also, AUC values for endocan and FLI were statistically similar $(p=0.594)$. In other words, prediction power of endocan for fatty liver is similar with FLI and HSI. However, FLI predictive power for fatty liver is more than HSI $(p=0.048)$ (Table 4$)$.

\section{Discussion}

In this present study, we demonstrated low serum concentrations of endocan in patients with metabolic syndrome with NAFLD in comparison with healthy individuals, which gives an insight into the role of endocan in NAFLD.

Patients with metabolic syndrome are prone to cardiovascular complications more frequently than the healthy population due to visceral obesity, defects in glucose metabolism, and endothelial dysfunction [17]. Recently, NAFLD was regarded as the hepatic manifestation of metabolic syndrome; however, it is still speculative as to which produces the latter [18]. Consequently, both NAFLD and metabolic syndrome have emerged as a growing health problem in developed countries [19]. Although the main trigger factor is insulin resistance in relation with metabolic syndrome, a genetic predisposition and unhealthy lifestyle can precipitate the development of fatty liver [20]. Other than insulin resistance, one of the key mechanisms recently mentioned is the endothelial dysfunction in the progression of NAFLD. It would seem that progression of NAFLD related to increase of active forms of oxygen and nitrogen which leads to a significant arterial vasospasm and a progressive damage to the endothelium [21]. Therefore, the cardiovascular consequences and related metabolic disorders require a multidisciplinary approach to the management of NAFLD.

NAFLD has a silent progress, and the majority of cases have an asymptomatic increase in AST and ALT. In general, diagnosis is suspected with abnormal liver function tests. Unfortunately, aminotransferases do not identify progressive disease. Ultrasonography, computed tomography, and magnetic resonance imaging of the liver are the standard imaging modalities used in clinical practice for diagnosis and detection disease progression [22]. Traditionally, liver biopsy is the gold standard for the diagnosis and staging of NAFLD; however, it is impractical to perform and hard to convince patients for the procedure [23]. Recent studies suggested that with the use of standardized measurements, it was possible to detect amounts of steatosis as little as 10\% [24-26]. Besides radiologic evaluations, some indexes were developed using anthropometric and laboratory parameters in order to predict hepatosteatosis severity [27]. In our study, we found higher scores of FLI and HSI in patients with NAFLD than in healthy individuals.

Endocan, which is one of the endothelium-derived proteoglycans, has been suggested to be an indicator of endothelial activation in recent studies [28]. It has been shown to increase in patients with infectious disease, malignancy, cardiovascular disease, and type 2 diabetes [29-32]. In a study by Dallio et al., NAFLD was found to be associated with a significant increase in serum endocan levels when compared with healthy controls. However, it was later realized that this increase was more marked in steatohepatitis than in simple steatosis, independent from the presence of diabetes [33]. In our study, the mean level of serum endocan in healthy individuals was $433.71 \mathrm{pg} / \mathrm{mL}$, similar to previous studies [34-36]. We found low serum endocan levels in patients with metabolic syndrome with fatty liver disease, which seems to conflict with some other studies [36]. However, due to lack of liver biopsy of the patient group, we could not demonstrate the ratio of steatohepatitis, which is more likely to be associated with endothelial dysfunction among patients with NAFLD.

In a similar manner, Ustyol et al. found that NAFLD had no additional influence on circulating serum endocan concentrations in adolescents with obesity, which is in accord with the findings of Janke et al. [28]. Janke et al. hypothesized that due to loss of a vasoprotective factor, obesity could be associated with decreased ESM-1 formation in adipose tissue and decreased endocan levels in plasma [37]. These data obtained from previous studies can clarify the negative correlation of the serum endocan levels and BMI of the patients in our study. The other parameter that showed a correlation with endocan was age. According to our results and those 
TABLe 3: ROC analysis and diagnostic screening tests were used to determine the cutoff point for endocan, HSI, and FLI.

\begin{tabular}{lccccccc}
\hline Steatosis present vs. not present & AUC & $p$ value & Cutoff & Sensitivity & Specificity & PPV+ & PPV- \\
\hline Endocan & 0.867 & $<0.001$ & 122.583 & 71.79 & 90.0 & 93.3 & 62.1 \\
HSI & 0.814 & $<0.001$ & $>16.67$ & 92.3 & 60.00 & 81.8 & 80.00 \\
FLI & 0.893 & $<0.001$ & $>40.86$ & 73.7 & 100.00 & 100.00 & 54.5 \\
\hline
\end{tabular}

ROC: receiver operating system; HSI: hepatic steatosis index; FLI: fatty liver index; AUC: area under curve. Statistical significance: $p<0.05$ and $p<0.01$.

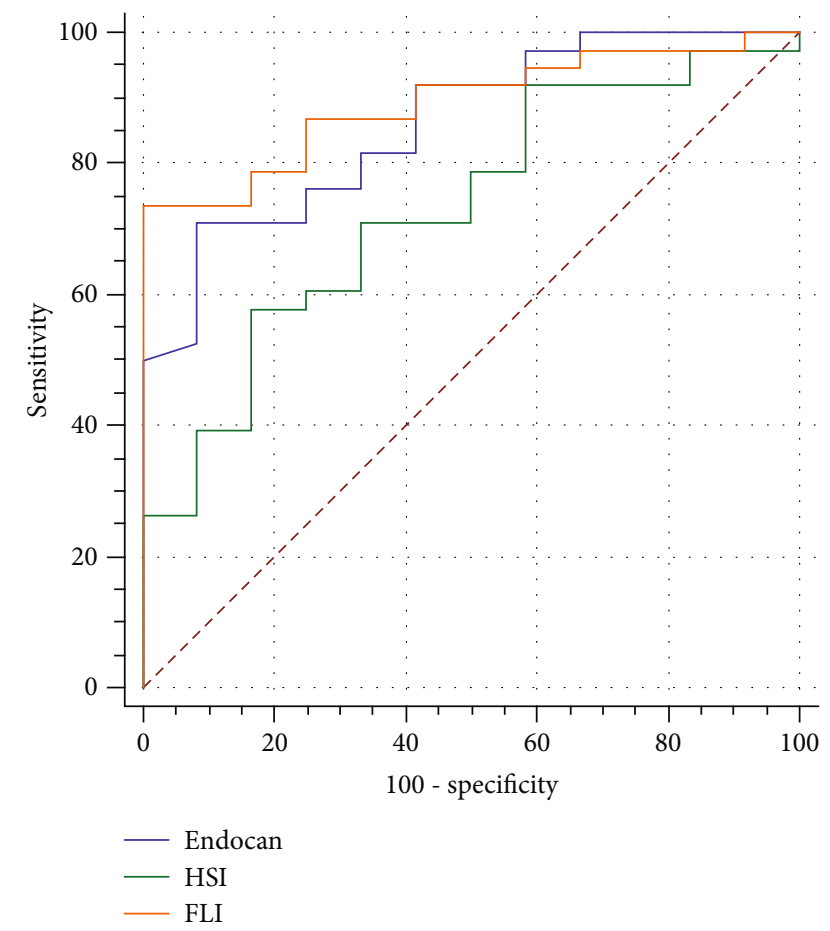

FIGURE 1: Sensitivity and specificity parameters for serum endocan, HSI, and FLI in patients of NAFLD. HSI: hepatic steatosis index; FLI: fatty liver index; NAFLD: nonalcoholic fatty liver disease.

TABLE 4: The comparative assessment of AUC for endocan, HSI, and FLI.

\begin{tabular}{lcc}
\hline & HSI (AUC difference) & FLI (AUC difference) \\
& $p$ value & $p$ value \\
\hline \multirow{2}{*}{ Endocan } & $(0.130)$ & $(0.031)$ \\
& 0.219 & 0.594 \\
HSI & & $(0.162)$ \\
& & 0.048 \\
\hline
\end{tabular}

HSI: hepatic steatosis index; FLI: fatty liver index; AUC: area under curve.

in previous studies, this positive correlation may be related with factors other than obesity.

To our knowledge, the relation between serum endocan and NAFLD has not yet been investigated in patients with metabolic syndrome. We demonstrated the predictive capacity of serum endocan regarding NAFLD in metabolic syndrome, with an AUROC of 0.867. For a patient with metabolic syndrome, serum endocan levels less than $122.583 \mathrm{pg} / \mathrm{mL}$ predict NAFLD with a sensitivity of $71.79 \%$ and a specificity of $90 \%$. It had a positive predictive value of $93.3 \%$ and a negative predictive value of $93.3 \%$ (Table 3 ). Along with these data, for a patient with metabolic syndrome, the cutoff value of FLI to predict NAFLD was 40.86, and the cutoff value of HSI was 16.76 to predict NAFLD which were similar to the literature data $[14,15]$. The comparison of serum endocan, HSI, and FLI in relation to predictive values, serum endocan was seem to have similar clinical value (Table 4). However, serum endocan gives an insight about endothelial dysfunction and inflammation in patients with NAFLD in metabolic syndrome which should be further addressed in clinical studies.

More importantly, the standardization and cutoff values of serum endocan have been controversial in the literature. For the first time, the current study suggests a cutoff point for serum endocan of NAFLD in patients with metabolic syndrome. However, there are some limitations of our study. The first limitation is the small sample size, which can serve as a pilot study. The second limitation is the lack of histologic confirmation of NAFLD; however, it is costly and impractical to perform in a disease with such a high prevalence. On the other hand, hepatic imaging is an acceptable assessment tool in NAFLD [7]. Finally, it has been suggested that the usefulness of FLI in mild hepatosteatosis is questionable, which may have led to underdiagnosis of fatty liver [15]. In our study, we used biochemical parameters, FLI, HSI, and ultrasound examinations in patients to increase the accuracy of the assessment of NAFLD severity.

In conclusion, NAFLD was predicted using SBP, BMI, waist circumference, fasting plasma glucose, HbA1C, TG, ALT, GGT, CRP, and serum endocan, along with the ultrasound images. Although serum endocan was found to lack a correlation with FLI and HSI, ROC analysis revealed a prediction of NAFLD at $122.583 \mathrm{pg} / \mathrm{mL}$ for the first time in metabolic syndrome. Nevertheless, it should be acknowledged that other novel markers beyond serum endocan will further clarify the prediction of NAFLD and the role of endothelial dysfunction-mediated metabolic complications in patients with NAFLD.

\section{Data Availability}

The data used to support the findings of this study are available from the corresponding author upon request.

\section{Conflicts of Interest}

All authors declare there is no conflict of interest in this study. 


\section{Authors' Contributions}

Banu Boyuk and Seher Irem Cetin are responsible for the study concept and design. Hande Erman and Engin Beydogan are responsible for the analysis and interpretation of the data. Hande Erman and Banu Boyuk are responsible for the drafting of the manuscript. Banu Boyuk is responsible for the statistical analysis.

\section{Acknowledgments}

We would like to thank Dr. Savas Guzel for his valuable help in the analysis of biochemical data.

\section{References}

[1] P. M. Graffy and P. J. Pickhardt, "Quantification of hepatic and visceral fat by $\mathrm{CT}$ and $\mathrm{MR}$ imaging: relevance to the obesity epidemic, metabolic syndrome and NAFLD," The British Journal of Radiology, vol. 89, no. 1062, p. 20151024, 2016.

[2] Z. M. Younossi, A. B. Koenig, D. Abdelatif, Y. Fazel, L. Henry, and M. Wymer, "Global epidemiology of nonalcoholic fatty liver disease-meta-analytic assessment of prevalence, incidence, and outcomes," Hepatology, vol. 64, no. 1, pp. 73-84, 2016.

[3] J. P. Ong, A. Pitts, and Z. M. Younossi, "Increased overall mortality and liver-related mortality in non-alcoholic fatty liver disease," Journal of Hepatology, vol. 49, no. 4, pp. 608-612, 2008.

[4] A. K. Loomis, S. Kabadi, D. Preiss et al., "Body mass index and risk of nonalcoholic fatty liver disease: two electronic health record prospective studies," The Journal of Clinical Endocrinology, vol. 101, no. 3, pp. 945-952, 2016.

[5] T. Miyake, T. Kumagi, S. Furukawa et al., "Non-alcoholic fatty liver disease: factors associated with its presence and onset," Journal of Gastroenterology and Hepatology, vol. 28 Suppl 4, pp. 71-78, 2013, Review.

[6] K. G. M. M. Alberti, R. H. Eckel, S. M. Grundy et al., "Harmonizing the Metabolic Syndrome," Circulation, vol. 120, no. 16, pp. 1640-1645, 2009.

[7] N. Chalasani, Z. Younossi, J. E. Lavine et al., "The diagnosis and management of nonalcoholic fatty liver disease: practice guidance from the American Association for the Study of Liver Diseases," Hepatology, vol. 67, no. 1, pp. 328-357, 2012.

[8] A. Tannapfel, H. Denk, H. P. Dienes et al., "Histopathological diagnosis of non-alcoholic and alcoholic fatty liver disease," Virchows Archiv, vol. 458, no. 5, pp. 511-523, 2011.

[9] F. Nascimbeni, S. Ballestri, M. V. Machado et al., "Clinical relevance of liver histopathology and different histological classifications of NASH in adults," Expert Review of Gastroenterology \& Hepatology, vol. 12, no. 4, pp. 351-367, 2018.

[10] S. Sarrazin, E. Adam, M. Lyon et al., "Endocan or endothelial cell specific molecule-1 (ESM-1): a potential novel endothelial cell marker and a new target for cancer therapy," Biochimica et Biophysica Acta, vol. 1765, no. 1, pp. 25-37, 2006.

[11] S. Balta, D. P. Mikhailidis, S. Demirkol, C. Ozturk, T. Celik, and A. Iyisoy, "Endocan: A novel inflammatory indicator in cardiovascular disease?," Angiology, vol. 243, no. 1, pp. 339$343,2015$.
[12] M. R. Ajmal, M. Yaccha, M. A. Malik et al., "Prevalence of nonalcoholic fatty liver disease (NAFLD) in patients of cardiovascular diseases and its association with hs-CRP and TNF- $\alpha$," Indian Heart Journal, vol. 66, no. 6, pp. 574-579, 2014.

[13] National Cholesterol Education Program (NCEP) Expert Panel on Detection, "Evaluation, and treatment of high blood cholesterol in adults (adult treatment panel III) final report," Circulation, vol. 106, pp. 3143-3421, 2002.

[14] J. H. Lee, D. Kim, H. J. Kim et al., "Hepatic steatosis index: a simple screening tool reflecting nonalcoholic fatty liver disease," Digestive and Liver Disease, vol. 42, no. 7, pp. 503-508, 2010.

[15] G. Bedogni, S. Bellentani, L. Miglioli et al., "The fatty liver index: a simple and accurate predictor of hepatic steatosis in the general population," BMC Gastroenterology, vol. 6, no. 1, p. 33, 2006.

[16] A. J. Sanyal and American Gastroenterological Association, "AGA technical review on nonalcoholic fatty liver disease," Gastroenterology, vol. 123, no. 5, pp. 1705-1725, 2002.

[17] K. Yamagata, "Do coffee polyphenols have a preventive action on metabolic syndrome associated endothelial dysfunctions? An assessment of the current evidence," Antioxidants, vol. 7, no. 2, p. 26, 2018.

[18] P. Almeda-Valdés, D. Cuevas-Ramos, and C. A. Aguilar-Salinas, "Metabolic syndrome and non-alcoholic fatty liver disease," Annals of Hepatology, vol. 8, no. 1, pp. 18-24, 2009.

[19] B. J. Perumpail, M. A. Khan, E. R. Yoo, G. Cholankeril, D. Kim, and A. Ahmed, "Clinical epidemiology and disease burden of nonalcoholic fatty liver disease," World Journal of Gastroenterology, vol. 23, no. 47, pp. 8263-8276, 2017.

[20] L. Abenavoli, L. Boccuto, A. Federico et al., "Diet and nonalcoholic fatty liver disease: the Mediterranean way," International Journal of Environmental Research and Public Health, vol. 16, no. 17, p. 3011, 2019.

[21] O. S. Khukhlina, A. A. Antoniv, O. Y. Mandryk, V. S. Smandych, and M. R. Matushchak, "The role of endothelial dysfunction in the progression mechanisms of non-alcoholic steatohepatitis in patients with obesity and chronic kidney disease," Wiadomości Lekarskie, vol. 72, no. 4, pp. 523526, 2019.

[22] J. M. Pappachan, S. Babu, B. Krishnan, and N. C. Ravindran, "Non-alcoholic fatty liver disease: a clinical update," Journal of Clinical and Translational Hepatology, vol. 5, no. 4, pp. 384-393, 2017.

[23] R. Jamali, A. Arj, M. Razavizade, and M. H. Aarabi, "Prediction of nonalcoholic fatty liver disease via a novel panel of serum adipokines," Medicine, vol. 95, no. 5, p. e2630, 2016.

[24] S. Ballestri, F. Nascimbeni, E. Baldelli et al., "Ultrasonographic fatty liver indicator detects mild steatosis and correlates with metabolic/histological parameters in various liver diseases," Metabolism, vol. 72, pp. 57-65, 2017.

[25] S. Ballestri, A. Lonardo, D. Romagnoli et al., "Ultrasonographic fatty liver indicator, a novel score which rules out NASH and is correlated with metabolic parameters in NAFLD," Liver International, vol. 32, no. 8, pp. 1242-1252, 2012.

[26] S. Ballestri, D. Romagnoli, F. Nascimbeni, G. Francica, and A. Lonardo, "Role of ultrasound in the diagnosis and treatment of nonalcoholic fatty liver disease and its complications," Expert Review of Gastroenterology \& Hepatology, vol. 9, no. 5, pp. 603-627, 2015. 
[27] J. K. Dowman, J. W. Tomlinson, and P. N. Newsome, "Systematic review: the diagnosis and staging of non-alcoholic fatty liver disease and non-alcoholic steatohepatitis," Alimentary Pharmacology \& Therapeutics, vol. 33, no. 5, pp. 525-540, 2010.

[28] A. Ustyol, E. Aycan Ustyol, F. Gurdol, F. Kokali, and S. Bekpinar, "P-selectin, endocan, and some adhesion molecules in obese children and adolescents with non-alcoholic fatty liver disease," Scandinavian Journal of Clinical and Laboratory Investigation, vol. 77, no. 3, pp. 205-209, 2017.

[29] N. D. F. Caires, A. Gaudet, L. Portier, A. Tsicopoulos, D. Mathieu, and P. Lassalle, "Endocan, sepsis, pneumonia, and acute respiratory distress syndrome," Critical Care, vol. 22, no. 1, p. 280, 2018.

[30] K. H. Kim, H. H. Lee, Y. E. Yoon et al., "Clinical validation of serum endocan (ESM-1) as a potential biomarker in patients with renal cell carcinoma," Oncotarget, vol. 9, no. 1, pp. 662667, 2017.

[31] T. Zhao, Y. Kecheng, X. Zhao et al., “The higher serum endocan levels may be a risk factor for the onset of cardiovascular disease: a meta-analysis," Medicine, vol. 97, no. 49, p. e13407, 2018.

[32] I. Balamir, I. Ates, C. Topcuoglu, and T. Turhan, “Association of endocan, ischemia-modified albumin, and hsCRP levels with endothelial dysfunction in type 2 diabetes mellitus," Angiology, vol. 69, no. 7, pp. 609-616, 2018.

[33] M. Dallio, M. Masarone, G. G. Caprio et al., "Endocan serum levels in patients with non-alcoholic fatty liver disease with or without type 2 diabetes mellitus: a pilot study," Journal of Gastrointestinal and Liver Diseases, vol. 26, no. 3, pp. 261268, 2017.

[34] D. Bechard, V. Meignin, A. Scherpereel et al., "Characterization of the secreted form of endothelial-cell-specific molecule 1 by specific monoclonal antibodies," Journal of Vascular Research, vol. 37, no. 5, pp. 417-425, 2000.

[35] B. D. Grigoriu, F. Depontieu, A. Scherpereel et al., "Endocan expression and relationship with survival in human nonsmall cell lung cancer," Clinical Cancer Research, vol. 12, no. 15, pp. 4575-4582, 2006.

[36] K. Ozaki, N. Toshikuni, J. George et al., "Serum endocan as a novel prognostic biomarker in patients with hepatocellular carcinoma," Journal of Cancer, vol. 5, no. 3, pp. 221-230, 2014.

[37] J. Janke, S. Engeli, K. Gorzelniak et al., “Adipose tissue and circulating endothelial cell specific molecule-1 in human obesity," Hormone and Metabolic Research, vol. 38, no. 1, pp. 28-33, 2006. 